\title{
Inhibitory effects of pomegranate flower extract and vitamin B3 on the formation of acrylamide during the donut making process
}

\author{
Mahin Heydari Ashkezari ${ }^{1} \cdot$ Mania Salehifar ${ }^{1}$
}

Received: 8 March 2018 / Accepted: 22 November 2018 / Published online: 3 December 2018

(c) The Author(s) 2018

\begin{abstract}
The aim of this study was to investigate the effects of pomegranate flower extract (PFE) $(0.05,0.04$, and $0.07 \%)$ and vitamin $\mathrm{B}_{3}(1.5,1.9$, and $2.3 \%)$ on the formation of acrylamide during the donut making process. Physicochemical, textural and sensory attributes and also acrylamide content of donuts were evaluated. Results showed that the addition of PFE and vitamin $B_{3}$ decreased acrylamide content, peroxide value, thiobarbituric acid value, oil adsorption capacity and specific volume and increased hardness of donuts. PFE incorporation declined $b^{*}$ and $L^{*}$ values, while vitamin $\mathrm{B}_{3}$ increased $\mathrm{L}^{*}$ index. All sensory features declined as the levels of PFE and vitamin $\mathrm{B}_{3}$ increased. Based on the optimization process, fried donuts containing $0.07 \%$ PFE and $1.97 \%$ vitamin $\mathrm{B}_{3}$ had the closest attributes to control sample and were accepted as the best treatment. In optimized donut, acrylamide content decreased from 76.2 to $64.4 \mathrm{mg} \mathrm{kg}^{-1}$ ( $11.8 \%$ reduction) upon incorporation.
\end{abstract}

Keywords Acrylamide - Donut · Pomegranate flower extract · Vitamin $\mathrm{B}_{3} \cdot$ Physicochemical properties

\section{Introduction}

Cereal products like sweet cakes, pies, and donuts are consumed as quick snacks all over the world. These products are an important source of energy and nutrients such as minerals, vitamins, and carbohydrates. Donuts are one of the most popular cereal products that are less widely considered in comparison to the main products. These cereal-based products have unique attributes such as attractive aroma and taste, but contain a significant amount of acrylamide $[1,2]$.

Acrylamide, a white crystalline solid, is classified as potentially carcinogenic compound to humans. It is found in carbohydrate-rich foods, which potato and baking products are responsible for about 50 and $20 \%$ of human exposure to acrylamide, respectively [3]. Recently, acrylamide has received a great deal of attention due to its potentially neurotoxic, genotoxic, and carcinogenic activities. Therefore, there are some inhibition strategies for reducing the formation of acrylamide in model and real food systems like the use of lower temperatures and times, raw materials containing

Mania Salehifar

salehifarmania@yahoo.com

1 Department of Food Science and Technology, Shahr-e-Qods Branch, Islamic Azad University, Tehran, Iran fewer precursors, and exogenous additives such as polyphenols, which are widely applied for this purpose [4].

Polyphenols are recognized as secondary metabolites in fruits and vegetables, which have some functionalities include antioxidant, antitumor, and anti-inflammation activities. They are also able to modulate the immune system and protect the retina and cardiovascular system [5]. Several studies have shown that plant extracts containing polyphenols are able to reduce the content of acrylamide in many food systems [6-8].

Pomegranate (Punica granatum L.) belongs to the family Punicaceae and has some positive roles like antidiabetic, antitumoral, antioxidant, antimicrobial, and anti-inflammatory effects [9]. Pomegranate flower extract (PFE) contains polyphenols (gallic and ellagic acids) and triterpenoids which have potentially antioxidant abilities [10]. Additionally, the inhibitory effect of water-soluble vitamins on acrylamide formation in fried potato strips has been reported; water-soluble vitamins such as $\mathrm{B}_{2}, \mathrm{~B}_{5}$, and $\mathrm{B}_{12}$ can reduce the synthesis of acrylamide by $20 \%$, which $B_{3}$ and $B_{6}$ vitamins, due to their strong antioxidant property and structural stability, are more effective in decreasing acrylamide formation during food processing. Furthermore, the active groups (primary amine, hydroxyl or aldehyde) of R-side chains can play an important role in the ability of vitamins to suppress the acrylamide formation [11]. Using PFE and vitamin $\mathrm{B}_{3}$ in donut formulation 
can lead to a positive effect on nutritional value and acrylamide reduction of the final product. In this regard, PFE can increase the antioxidant activity of the donut due to its polyphenolic compounds. Therefore, these bioactive compounds can be used as potential ingredients in order to inhibit the creation of acrylamide in thermally processed foods.

The aim of this study was, therefore, to evaluate the inhibitory effects of PFE as a source of polyphenols and vitamin $\mathrm{B}_{3}$ on the formation of acrylamide in donut during the making process.

\section{Materials and methods}

\section{Materials}

Pomegranate flowers were collected from Yazd province (Iran). All chemicals and reagents used in this study were of analytical grade and purchased from Merck (Darmstadt, Germany).

\section{Preparation of PFE}

Pomegranate flowers were completely dried at room temperature and then grounded to powder form. About $75 \mathrm{~g}$ of dried pomegranate flower was dispersed in $200 \mathrm{~mL}$ of $96 \%$ ethanol and the dispersion was heated at $45{ }^{\circ} \mathrm{C}$. The extract was transferred into a dark glass for $24 \mathrm{~h}$ and then filtered. Afterwards, the solvent was removed through Rotary evaporator (Buchi, Switzerland) to obtain an extract $(100 \mathrm{~mL})$ with $28.4 \%$ dry matter and then the extract (PFE) was stored at $4{ }^{\circ} \mathrm{C}$ until analyses.

\section{Preparation of donuts}

Donuts were prepared in accordance with the methods of Vélez-Ruiz and Sosa-Morales [12] and Shih et al. [13] with some modifications (Table 1). For this aim, all powder ingredients (flour, salt, sugar, yeast, vanilla, vitamin B3, and improver) were mixed for $2 \mathrm{~min}$. Afterwards, liquids like PFE, oil, egg, and water were added and the mixture was blended at low speed for 2 min followed by mixing at high speed for $7 \mathrm{~min}$. The doughs were cut into $50 \mathrm{~g}$ chins and proofed at $35^{\circ} \mathrm{C}$ for $45 \mathrm{~min}$. After, the chins with $1.0 \mathrm{~cm}$ thickness were spread manually, molded and proofed for $6 \mathrm{~min}$. They were then stored in an incubator for $70 \mathrm{~min}$ at $37{ }^{\circ} \mathrm{C}$ under $85 \%$ relative humidity to complete fermentation process. The doughs were fried at $180{ }^{\circ} \mathrm{C}$ for $2 \mathrm{~min}$ and then removed from the fryer. Finally, donuts were cooled down to room temperature for 45-60 min and stored in polyethylene bags at ambient temperature before uses.

\section{Donuts evaluation}

\section{Specific volume}

Specific volume of donuts was measured based on the rapeseed displacement AACC method 72-10 [14]. The weight of donuts was determined and then the specific volume was calculated as bellow:

Specific volume $\left(\mathrm{cm}^{3} / \mathrm{g}\right)=$ sample volume $\left(\mathrm{cm}^{3}\right) /$ sample weight $(\mathrm{g})$

Table 1 Donut formulations

\begin{tabular}{|c|c|c|c|c|c|c|c|c|c|c|c|}
\hline \multirow[t]{2}{*}{ Formulation } & \multicolumn{11}{|c|}{ Ingredients } \\
\hline & Flour (\%) & Milk (\%) & Sugar $(\%)$ & $\mathrm{NaCl}(\%)$ & Dry yeast (\%) & $\operatorname{Egg}(\%)$ & Oil (\%) & Vanilla (\%) & Improver (\%) & PFE (\%) & $\begin{array}{l}\text { Vita- } \\
\text { min } B_{3} \\
(\%)\end{array}$ \\
\hline 1 (control) & 100 & 60 & 1.6 & 0.8 & 0.4 & 1.2 & 1.25 & 0.2 & 0.3 & 0 & 0 \\
\hline 2 & 100 & 60 & 1.6 & 0.8 & 0.4 & 1.2 & 1.25 & 0.2 & 0.3 & 0.055 & 1.9 \\
\hline 3 & 100 & 60 & 1.6 & 0.8 & 0.4 & 1.2 & 1.25 & 0.2 & 0.3 & 0.055 & 1.9 \\
\hline 4 & 100 & 60 & 1.6 & 0.8 & 0.4 & 1.2 & 1.25 & 0.2 & 0.3 & 0.04 & 1.9 \\
\hline 5 & 100 & 60 & 1.6 & 0.8 & 0.4 & 1.2 & 1.25 & 0.2 & 0.3 & 0.04 & 2.3 \\
\hline 6 & 100 & 60 & 1.6 & 0.8 & 0.4 & 1.2 & 1.25 & 0.2 & 0.3 & 0.055 & 1.5 \\
\hline 7 & 100 & 60 & 1.6 & 0.8 & 0.4 & 1.2 & 1.25 & 0.2 & 0.3 & 0.055 & 1.9 \\
\hline 8 & 100 & 60 & 1.6 & 0.8 & 0.4 & 1.2 & 1.25 & 0.2 & 0.3 & 0.07 & 1.9 \\
\hline 9 & 100 & 60 & 1.6 & 0.8 & 0.4 & 1.2 & 1.25 & 0.2 & 0.3 & 0.055 & 1.9 \\
\hline 10 & 100 & 60 & 1.6 & 0.8 & 0.4 & 1.2 & 1.25 & 0.2 & 0.3 & 0.04 & 1.5 \\
\hline 11 & 100 & 60 & 1.6 & 0.8 & 0.4 & 1.2 & 1.25 & 0.2 & 0.3 & 0.055 & 1.9 \\
\hline 12 & 100 & 60 & 1.6 & 0.8 & 0.4 & 1.2 & 1.25 & 0.2 & 0.3 & 0.055 & 2.3 \\
\hline 13 & 100 & 60 & 1.6 & 0.8 & 0.4 & 1.2 & 1.25 & 0.2 & 0.3 & 0.07 & 1.5 \\
\hline 14 & 100 & 60 & 1.6 & 0.8 & 0.4 & 1.2 & 1.25 & 0.2 & 0.3 & 0.07 & 2.3 \\
\hline
\end{tabular}




\section{Moisture content}

Moisture content of donuts was measured in accordance with the AACC method 44-14 [14] by drying the samples in an oven (Memmert unb 500, Germany) at $100-105{ }^{\circ} \mathrm{C}$ for $2 \mathrm{~h}$.

\section{Acrylamide measurement}

The amount of acrylamide in fried donuts was quantified by high performance liquid chromatography (HPLC) after derivatization based on the methods described by Shi et al. [15] and Jezussek and Schieberle [16]. Donut samples were transferred to the laboratory and after drying the samples at ambient temperature $\left(28^{\circ} \mathrm{C}\right)$, they were grounded to small particles (particle size up to $1.0 \mathrm{~mm}$ ) using an electric mill (Black \& daker, Germany) and then stored at $-4{ }^{\circ} \mathrm{C}$. In order to extract and measure the amount of acrylamide using a LC/DAD apparatus, 2-mercaptobenzoic acid was employed as an acrylamide derivative. $5.0 \mathrm{~g}$ of the milled donuts was accurately weighed and transferred to a centrifuge tube containing $25 \mathrm{~mL}$ of deionized water. The tube was well stirred for $5 \mathrm{~min}$ and then placed in an ultrasonic bath at $30{ }^{\circ} \mathrm{C}$ for 20 min to increase the extraction efficiency. Next, the tube was centrifuged at $3850 \mathrm{rpm}$ for $15 \mathrm{~min}$ at $0{ }^{\circ} \mathrm{C}$ and then $10.0 \mathrm{~mL}$ of supernatant was separated and transferred into another centrifuge tube. The solution was adjusted to $\mathrm{pH}$ 8.0 by adding $1.0 \mathrm{M} \mathrm{KOH}$. Then, 2-mercaptobenzoic acid $(1.0 \mathrm{~mL})$ was added to the solution in order to perform the derivative process ( $\mathrm{pH}$ value was increased to $\sim 10.0$ ). The tube was coated with aluminum foil and placed in an ultrasonic bath at $35{ }^{\circ} \mathrm{C}$ for $100 \mathrm{~min}$ to complete derivative reaction. Afterwards, $3.0 \mathrm{~mL}$ of saturated $\mathrm{Pb}\left(\mathrm{CH}_{3} \mathrm{COO}\right)_{2}$ was added to remove the excess of derivative reagent and after centrifugation at $3850 \mathrm{rpm}$ for $20 \mathrm{~min}$ at $10^{\circ} \mathrm{C}$, the supernatant was collected and transferred into another tube and was then acidified with $5.0 \mathrm{M} \mathrm{HCl}$ to $\mathrm{pH}$ value of 3.0. The tube was re-centrifuged and the supernatant was carefully separated and extracted with $5 \mathrm{~mL}$ of ethyl acetate (3-times). The organic solvent was evaporated at $60-65{ }^{\circ} \mathrm{C}$ and the residue was re-dissolved with $800 \mu \mathrm{L}$ of methanol. The sample was then collected and injected for HPLC analysis (Agilent, Germany). A mixture of acetonitrile and acetic acid solution $(0.1 \% \mathrm{w} / \mathrm{v})(20: 80 \mathrm{v} / \mathrm{v})$ was utilized as mobile phase. $20 \mu \mathrm{L}$ of sample was injected and flow rate was $7.0 \mathrm{~mL} \mathrm{~min}-1$. The detection wavelengths were set at 238 and $254 \mathrm{~nm}$.

\section{Peroxide value}

Peroxide value is used to determine the amount of initial oxidation products of fats and oils. Five grams of oil sample (palm-free frying oil) was weighed in $250 \mathrm{~mL}$ Erlenmeyer flask and $30 \mathrm{~mL}$ of peroxide solvent (acetic acid to chloroform ratio of 3:2) was added. The flask was shaken, $0.5 \mathrm{~mL}$ of saturated potassium iodine solution was added and then the obtain solution was placed in a dark place for $1.0 \mathrm{~min}$. After, $30 \mathrm{~mL}$ of distilled water was added and the liberated iodine was titrated with sodium thiosulfate in the presence of starch indicator. Peroxide value was calculated as milliequivalents of oxygen per kilogram of extracted oil (meq $/ \mathrm{kg}$ ) according to the following equation [17]:

Peroxide value : $[(\mathrm{N} \times \mathrm{V}) / \mathrm{W}] \times 1000$

where, $\mathrm{N}$ is the normality of sodium thiosulfate, $\mathrm{V}$ is the titrated volume of sodium thiosulfate, and $\mathrm{W}$ is the oil weight $(\mathrm{kg})$.

\section{Thiobarbituric acid (TBA) value}

Ten grams of sample was dissolved in $25 \mathrm{~mL}$ of distilled water and then diluted to $50 \mathrm{~mL}$ with trichloroacetic acid solution. The obtained solution was filtered and $4.0 \mathrm{~mL}$ of the filtrate was mixed with $1.0 \mathrm{~mL}$ of TBA solution $(0.06 \mathrm{M})$ followed by placing in a boiling water bath for $10 \mathrm{~min}$. Finally, the solution was cooled to room temperature and results were expressed as $\mathrm{mg}$ malonaldehyde (MDA) per kilogram [17].

\section{Oil adsorption capacity}

Oil adsorption capacity (OAC) was measured according to the standard AOAC method [18].

\section{Texture analysis}

Hardness of donuts was evaluated by a Texture Analyzer (M350-to act, UK) based on the AACC method 74-09 [14]. Donut samples were cut into pieces with $40 \mathrm{~mm} \times 40 \mathrm{~mm} \times 20 \mathrm{~mm}$ dimension and then the crust of donuts was removed. An aluminum cylindrical probe (25 mm diameter) was used to compress the samples at $30 \mathrm{~mm} \mathrm{~min}^{-1}$ test speed and maximum force $\left(\mathrm{F}_{\max }\right)$ was then recorded as hardness of the donuts. 


\section{Color studies}

The crust color ( $L, a$, and $b$ values) of donuts was evaluated using a Hunterlab colorimeter (HunterlabD25-Dp9000, USA) according to the AACC method 14-30 [14].

\section{Sensory attributes}

Donuts were evaluated for sensory attributes, i.e., flavor, aroma, texture, color, and overall acceptability by ten welltrained panelists based on a five-point hedonic scale test (score $1=$ dislike extremely and score $5=$ like extremely) [19].

\section{Statistical analysis}

Response surface methodology (RSM) was used to evaluate the effects of independent variables of the study and different levels of PFE and vitamin $B_{3}$ on physicochemical, textural and sensorial attributes of the donuts. The measured parameters were analyzed using the first, second, and three-degree polynomial equations. Data analysis was performed using Design Express 8.0 software.

\section{Results and discussion}

\section{Acrylamide content}

The result of analysis of variance (ANOVA) for acrylamide content is presented in Table 2. The model $F$ value of 33.68 and the $p$ value less than 0.0001 indicate that the model was significant. In addition, the $p$ values of linear coefficients (A for $\mathrm{PFE}$ and $\mathrm{B}$ for vitamin $\mathrm{B}_{3}$ ) and quadratic term coefficient ( $\mathrm{A}^{2}$ for PFE) were less than 0.05 . The coefficient of determination $\left(\mathrm{R}^{2}\right)$ and adjusted determination coefficient (adjusted $\mathrm{R}^{2}$ ) were 0.96 and 0.93 , which showed that the model is suitable for predicting the experimental data from acrylamide content of donuts. The addition of PFE and vitamin $\mathrm{B}_{3}$ caused a decrease of acrylamide content in donuts (Fig. 1a) mainly due to the antioxidant activities of the compounds thorough chelating of free radicals, scavenging of carbonyls, and limiting the degradation of sugars during the Maillard reaction [20]. Ciesarová et al. [21] reported a similar result and showed that the addition of plant extracts obtained from pimento, black pepper, marjoram, and oregano to potatoes decreased the content of acrylamide up to $75 \%$. Additionally, Budryn et al. [1] reported that high concentrations of the extracts of green tea and green coffee reduced the formation of acrylamide in fried donuts.

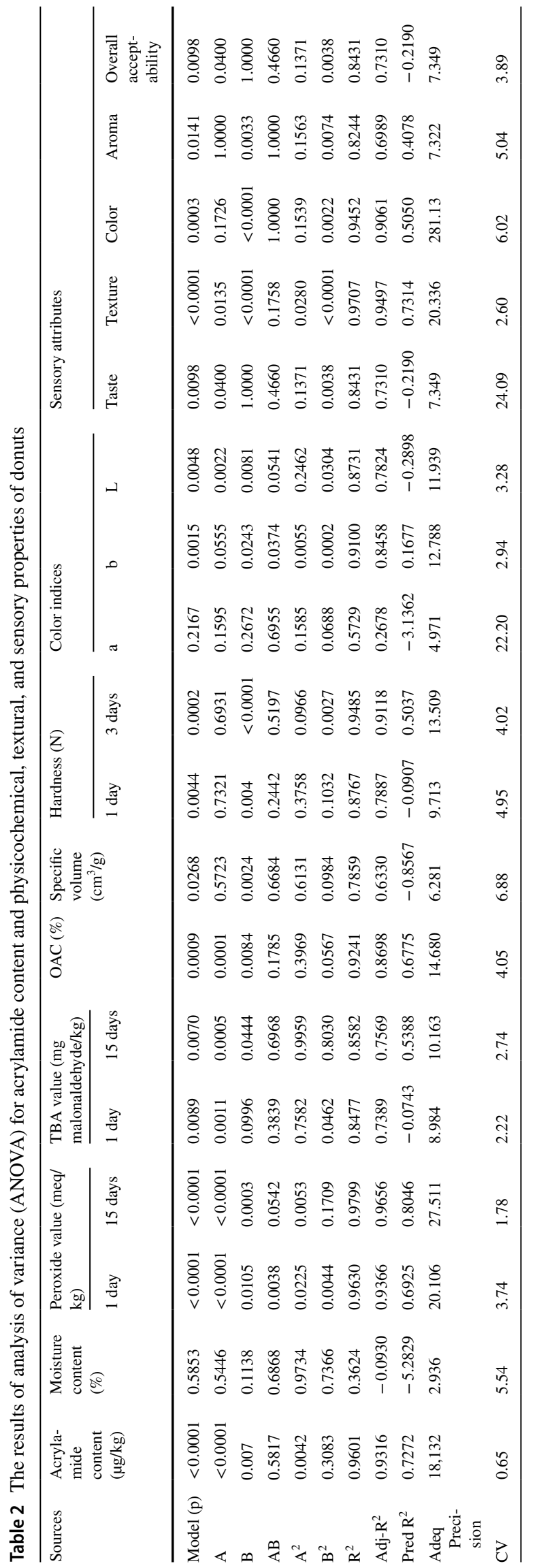



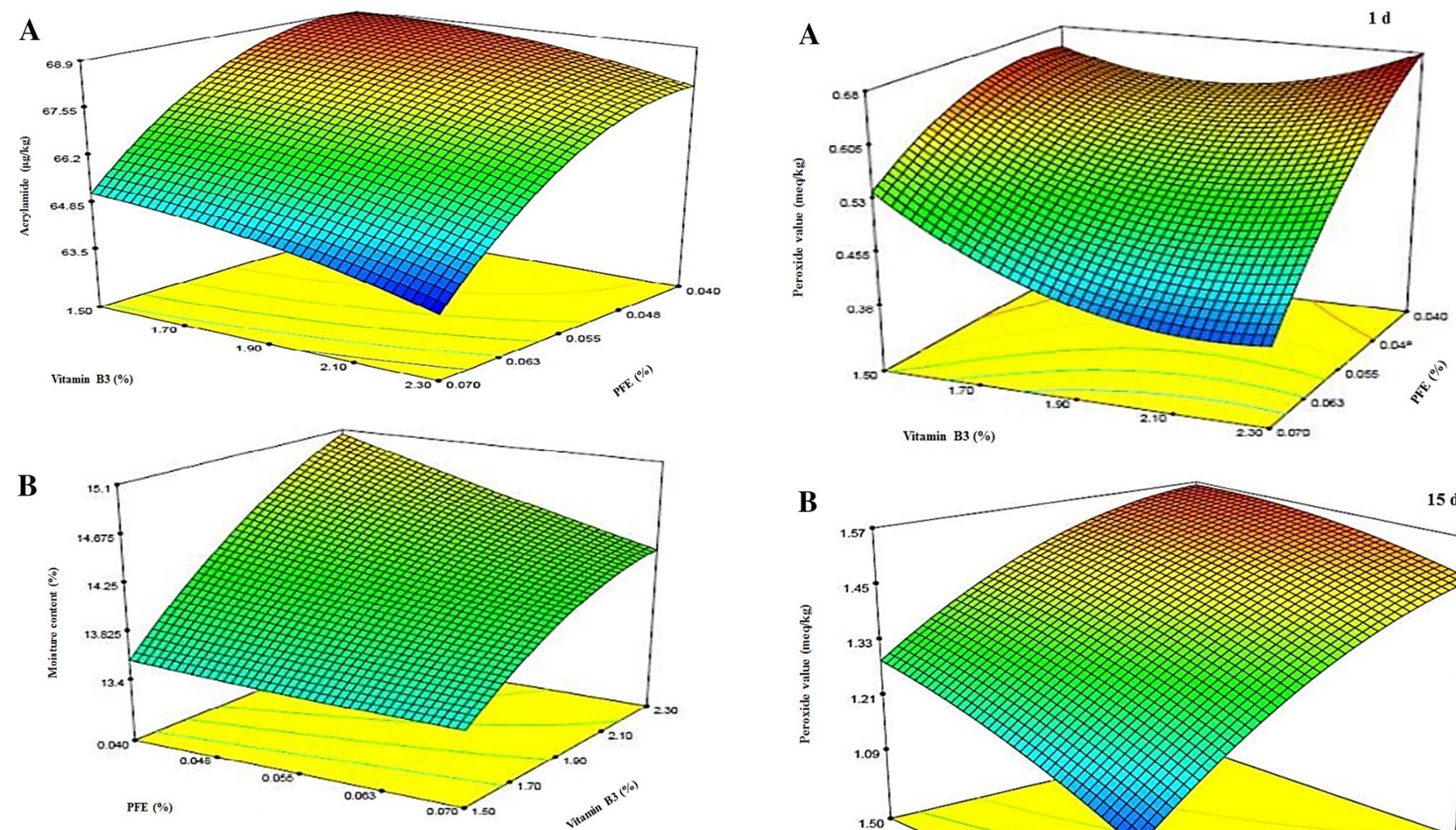

Fig. 1 Response surface plots for the effects of PFE and vitamin $B_{3}$ on acrylamide (a) and moisture (b) contents of donut

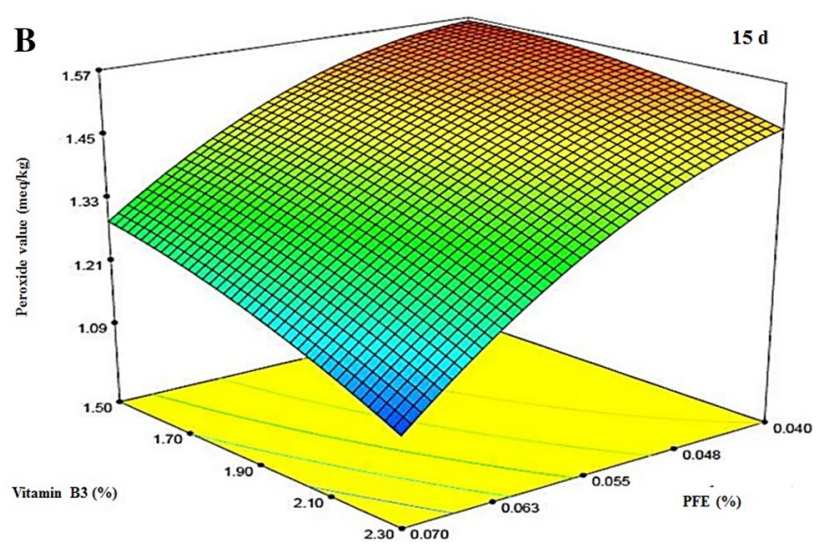

\section{Physicochemical properties}

The results of adding PFE and vitamin $\mathrm{B}_{3}$ to donut formulation and their effects on moisture content of the product are shown in Fig. $1 \mathrm{~b}$ and Table 2. Independent variables had no significant effect on the response. Incorporation of PFE and vitamin $\mathrm{B}_{3}$ had significant linear, quadratic, and interaction effects on peroxide value of the donuts after production (first day) (Table 2). As well, inverse linear and non-linear correlations were observed between PFE and vitamin $\mathrm{B}_{3}$ and peroxide value, respectively (Fig. 2a). Moreover, linear and interaction effects of independent variables and quadratic effect of PFE were significant in terms of peroxide value of the donut after 15 days of production (Table 2). Figure $2 b$ shows a linear and inverse relationship between independent variables and peroxide value. The lowest peroxide index was observed in donut samples containing high levels of PFE and vitamin $B_{3}$. Reduced peroxide value in samples treated with extract is mainly due to the presence of phenolic compounds, especially polyphenols (e.g., tannins) in pomegranate flowers $[22,23]$. As well, vitamin $B_{3}$ has antioxidant activity and is used as an effective agent to prevent oxidation reactions [11]. These results are in accordance with those of Izzreen and Noriham [24], who reported that the inclusion of some Malaysian herbal aqueous extracts into cake decreased peroxide value of the product after 15 days of production.

Fig. 2 Response surface plots for the effects of PFE and vitamin $B_{3}$ on peroxide value (a 1 day and $\mathbf{b} 15$ days) of donut

TBA value decreased linearly and non-linearly with increasing the levels of PFE and vitamin $\mathrm{B}_{3}$, respectively (Fig. 3a, b) (Table 2). The reactivity of TBA with lipids is an indicator of the extent of secondary oxidation of lipids in foods; an increase in that indicates the progression of the oxidation process and hydroxide degradation into volatile compounds, especially aldehydes [25]. The decreased amount of TBA value is due to the presence of antioxidant phenolic compounds (gallic acid and ellagic acid) in PFE [26] and potent antioxidant activity of vitamin $\mathrm{B}_{3}$ [11]. The results are in line with those of Bopitiya and Madhujith [27], who reported that pomegranate peel extract can effectively delay the oxidation process of coconut oil during frying.

As shown in Fig. 4a and Table 2, OAC of donuts declined linearly as PFE and vitamin $B_{3}$ levels increased. By-products of the oxidation reaction such as high molecular weight polar compounds are able to increase the oil viscosity and reduce the surface tension between oil and food product, which in turn, the oil can be readily adsorbed into the food [28]. Accordingly, the addition of PFE and vitamin $\mathrm{B}_{3}$ due their antioxidant capacities reduced OAC of donuts through inhibiting the oxidation process and formation of free radicals. Aydenız and Yilmaz [29] reported similar results. 

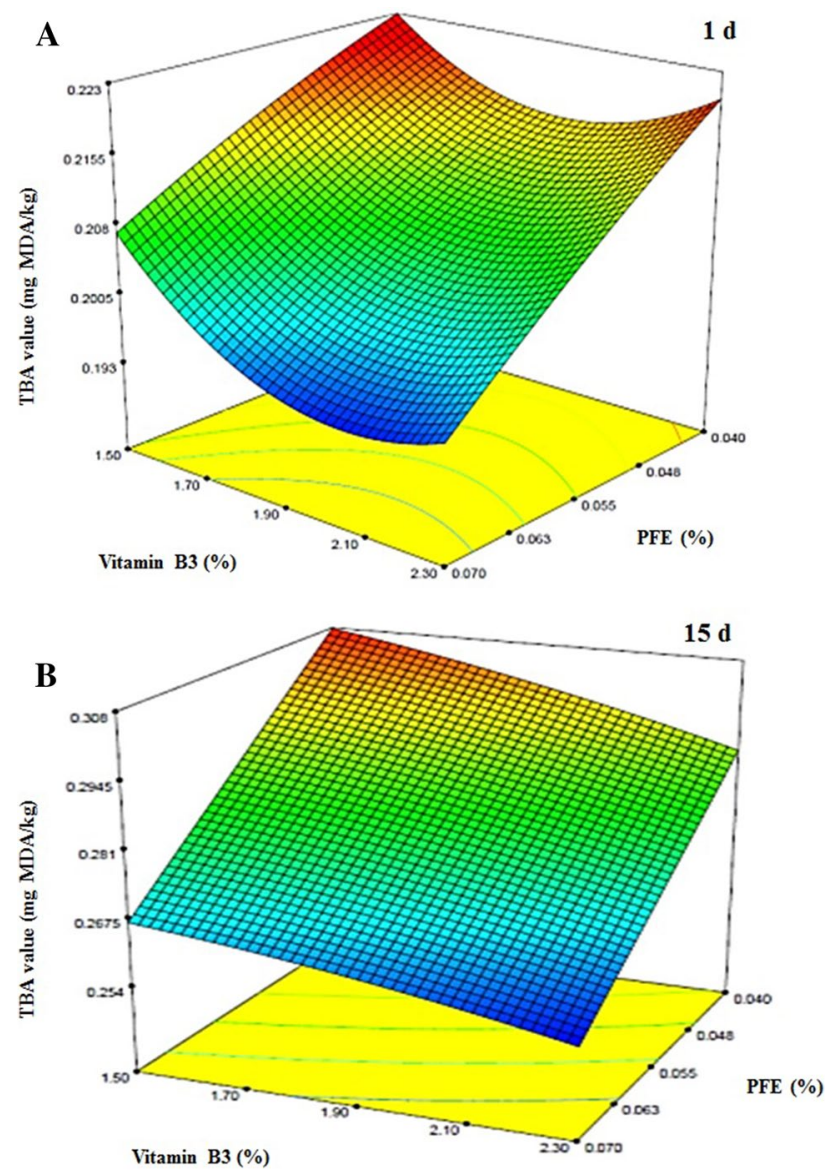

Fig. 3 Response surface plots for the effects of PFE and vitamin $B_{3}$ on TBA value (a 1 day and $\mathbf{b} 15$ days) of donut

ANOVA results for specific volume (Table 2) showed that the proposed model was significant $(\mathrm{P}<0.05)$. The only significant expression of the model was the effect of vitamin $\mathrm{B}_{3}$ (B) $(\mathrm{P}<0.05)$. Additionally, the counter plot (Fig. 4b) shows that there is an inverse and non-linear correlation between vitamin $B_{3}$ and specific volume. The specific volume decreased as the level of vitamin $\mathrm{B}_{3}$ increased, while $\mathrm{PFE}$ had no significant effect $(\mathrm{P}>0.05)$.

\section{Textural attributes}

According to the Table 2, the model obtained for the effects of vitamin $B_{3}$ and PFE on textural attributes of donuts was significant in terms of vitamin $B_{3}$ at 1 and 15 days, while the effect of PFE was not significant. On the first day, donut hardness increased linearly with increasing vitamin $\mathrm{B}_{3}$ levels (Fig. 5a). Among the expressions, the effect of vitamin $\mathrm{B}_{3}(\mathrm{~B})$ was significant $(\mathrm{P}<0.05)$. The statistical analysis of the effect of independent variables on donut texture after 15 days of production showed that the
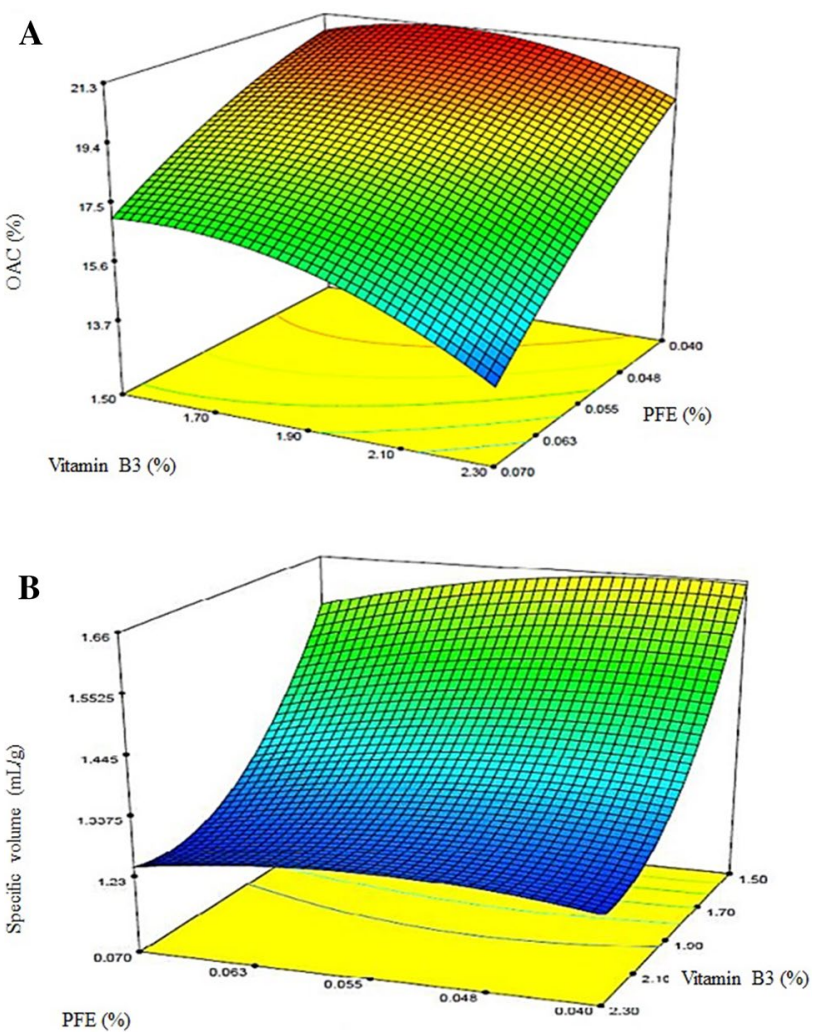

Fig. 4 Response surface plots for the effects of PFE and vitamin $B_{3}$ on donut OAC (a) and specific volume (b)

effect of vitamin $B_{3}(B)$ and quadratic effect of vitamin $B_{3}$ $\left(\mathrm{B}^{2}\right)$ were statistically significant (Table 2$)$. A non-linear effect was observed between vitamin $\mathrm{B}_{3}$ levels and donut hardness (Fig. 5b); higher levels of vitamin $\mathrm{B}_{3}$ resulted in an increase in hardness of donuts. The decreased specific volume (increased hardness) of donuts could be attributed to the adverse effect of the vitamin on the formation of gluten network, which the latter is not able to retain gas bubbles and, in turn, leads to a reduction in volume and a rigid and compact texture [30-32]. Wang et al. [33] reported that the addition of green tea extracts into dough bread lowered the porosity and increased the hardness and adhesiveness of the final product.

\section{Donut color}

The influence of vitamin $\mathrm{B}_{3}$ and PFE on $\mathrm{a}^{*}$ value is shown in Table 2 and Fig. 6a. The statistical analysis showed that the independent variables had no significant effect on $\mathrm{a}^{*}$ value at a confidence level of $95 \%$ (Table 2). The results of the present study are in line with those of Sheikholeslami et al. [34]. 
A

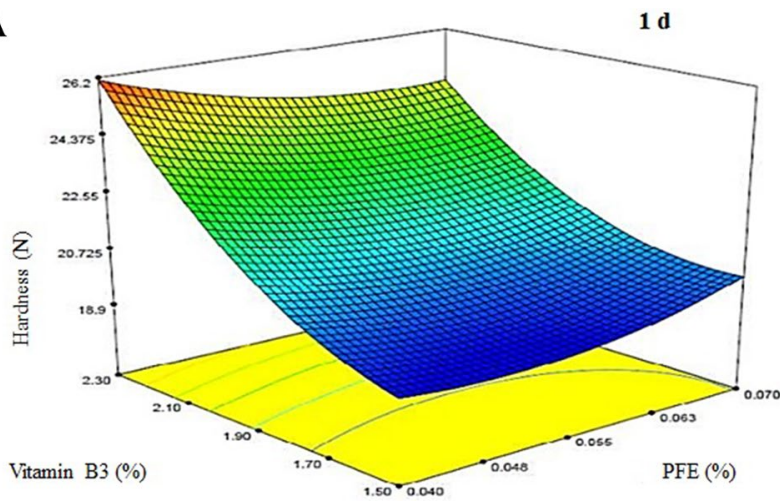

B

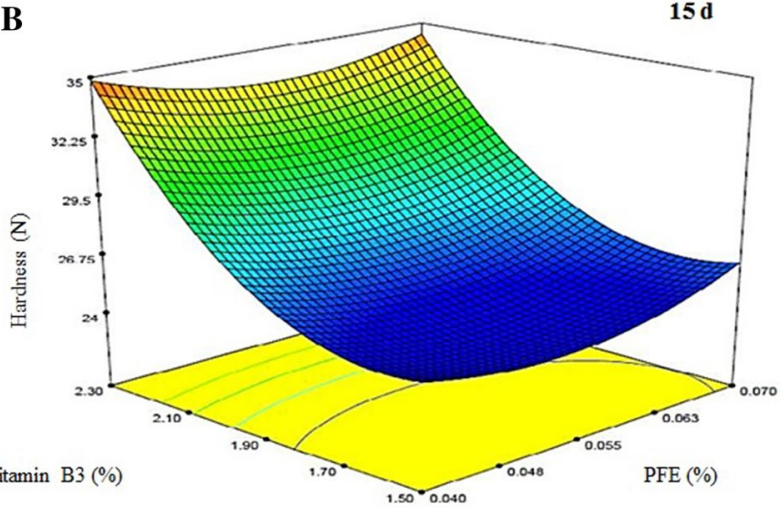

Fig. 5 Response surface plots for the effects of PFE and vitamin $B_{3}$ on donut hardness (a 1 day and $\mathbf{b} 15$ days)

In terms of $b^{*}$ value, the effect of PFE (A), vitamin $B_{3}$ (B), interaction effect $(A B)$, quadratic effects of PFE $\left(A^{2}\right)$ and vitamin $\mathrm{B}_{3}\left(\mathrm{~B}^{2}\right)$ were significant $(\mathrm{P}<0.05)$ (Table 2$)$. According to Fig. 6b, there is a non-linear relationship between PFE and $b^{*}$ index, which means that $b^{*}$ value decreases with increasing level of PFE. Donuts containing vitamin $B_{3}$, as shown in Fig. 6b, had a similar trend in $b^{*}$ index. The reduction of $b^{*}$ value can be attributed to chemical reactions, especially browning ones. The increased lightness of donuts is mainly due to the presence of vitamin $B_{3}$, as donuts contains very low level of PFE. The addition of vitamin $B_{3}$ resulted in decrease in the extent of the Maillard reaction probably because of its water absorption capacity, which in turn led to a decrease in $b^{*}$ index. Similar results were reported by Ajila et al. [31], who stated that the addition of mango peel powder to biscuit formulation led to a decrease in $b^{*}$ value of the product due to enzymatic browning derived from peroxidase and polyphenol oxidase existed in mango peel.

The results of ANOVA for $\mathrm{L}^{*}$ index demonstrated that the effect of PFE (A), vitamin $B_{3}(B)$, interaction effect $(A B)$, and quadratic effect of vitamin $B_{3}\left(B^{2}\right)$ were significant $(\mathrm{P}<0.05)($ Table 2). Figure $6 \mathrm{c}$ depicts that there is a
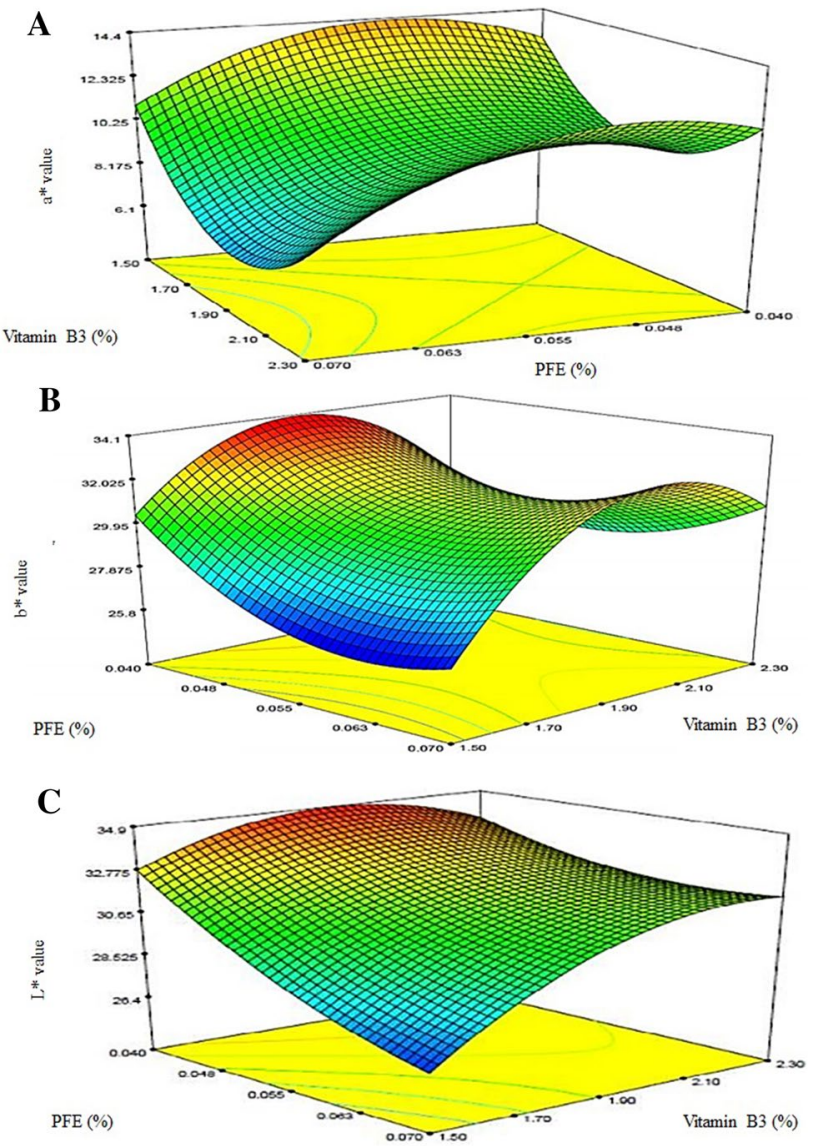

Fig. 6 Response surface plots for the effects of PFE and vitamin $B_{3}$ on $\mathrm{a}^{*}$ value $(\mathbf{a}), \mathrm{b}^{*}$ value $(\mathbf{b})$, and $\mathrm{L}^{*}$ value $(\mathbf{c})$

reverse and non-linear relationship between PFE and $\mathrm{L}^{*}$ value and donuts containing higher levels of PFE showed lower L* index likely due to browning reactions in the presence of phenolic compounds, which reduced the lightness of samples. A direct and nonlinear correlation was observed between vitamin $B_{3}$ and $L^{*}$ value and the lightness of donuts increased as a function of vitamin level because of water absorption capacity of the vitamin as mentioned previously. The results are in agreement with the findings of Wang et al. [33] and Lu et al. [35], who reported that inclusion of green tea powder and green tea extract into cake and bread, respectively, decreased the $\mathrm{L}^{*}$ value, while the parameter increased in the presence of ascorbic acid.

\section{Sensory attributes}

The results showed that $\mathrm{PFE}$ and vitamin $\mathrm{B}_{3}$ had significant linear effect and quadratic effect on donut taste, respectively (Table 2). There was an inverse and non-linear relationship between PFE and vitamin $B_{3}$ in terms of taste (Fig. 7a). The response decreased as amount of PFE and vitamin $B_{3}$ 
increased and the highest taste score was obtained in donut samples containing low levels of independent variables. The decreased taste score of donuts made from high levels of PFE could be related to the presence of ellagic acid, which has bitter taste [10] and it can reduce the donut taste.

The independent variables had significant linear and quadratic effects on texture of donuts (Table 2). Donut samples made from low levels of PFE and vitamin $B_{3}$ had the highest texture score. As can be seen from Fig. 7b, the PFE addition rises and the vitamin $B_{3}$ addition decreases texture of donuts due to the negative effect of vitamin $\mathrm{B}_{3}$ on gluten formation [31]. A significant linear effect and also significant quadratic effect of vitamin $\mathrm{B}_{3}$ on donut color was observed, while the addition of PFE had no significant effect on this response (Table 2). Donuts containing low amount of vitamin $\mathrm{B}_{3}$ showed the highest color score. Figure 8 a shows that donut color decreases non-linearly as the level of vitamin $B_{3}$ is increased. Li et al. [36] reported that cookies enriched with $0.2 \mathrm{~g} \mathrm{~kg}^{-1}$ bamboo leaf antioxidants and $0.1 \mathrm{~g} \mathrm{~kg}^{-1}$ vitamin $\mathrm{E}$ showed similar sensory attributes (taste, color, and texture) compared to the control cookie.

The results of ANOVA for donut aroma showed significant linear and quadratic effects of vitamin $\mathrm{B}_{3}$ on this response whereas the effect of PFE was not significant (Table 2). Low levels of the independent variables resulted in a donut with the highest aroma score. According to
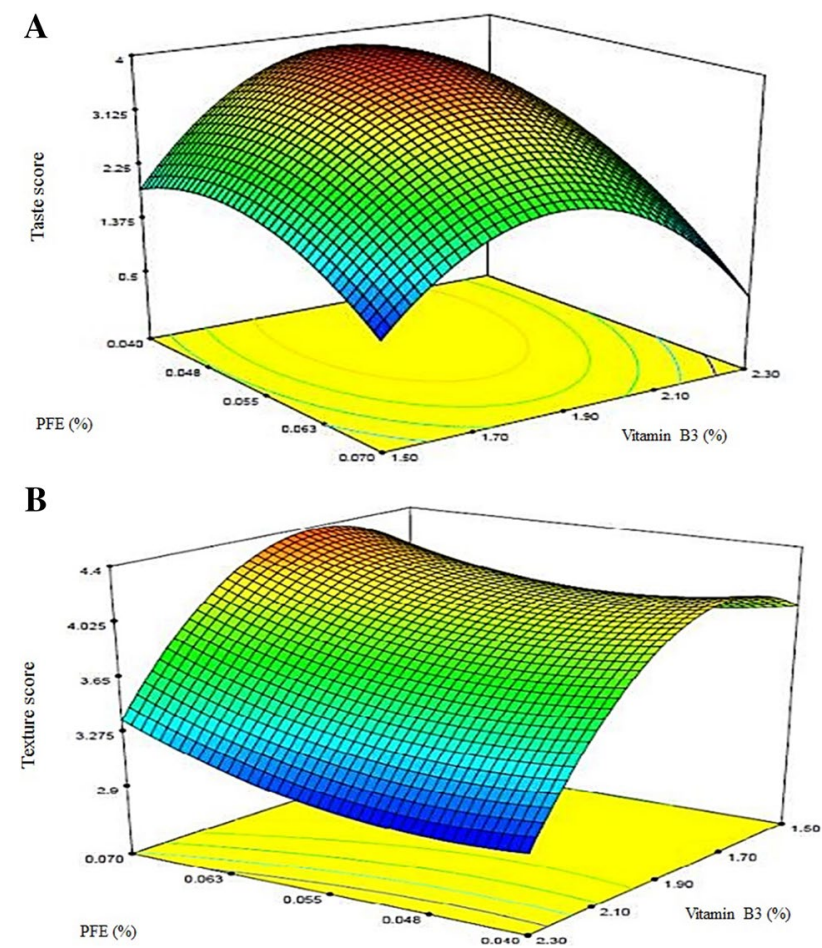

Fig. 7 Response surface plots for the effects of PFE and vitamin $B_{3}$ on donut taste (a) and texture (b)
Fig. $8 \mathrm{~b}$, there is a non-linear relationship between vitamin $\mathrm{B}_{3}$ and donut aroma and the response decreased as a function of vitamin $\mathrm{B}_{3}$ level. There were a linear effect of PFE and quadratic effect of vitamin $B_{3}$ on overall acceptability of donut samples (Table 2). Moreover, samples made from low levels of the independent variables presented higher overall acceptability. Additionally, the general acceptance of donuts declined with an increase in PFE and increased with increasing the level of vitamin $\mathrm{B}_{3}$ up to a certain point, followed by reduction with a further increase in the variable (Fig. 9).

\section{Optimization process}

The numerical optimization was applied to optimize values of independent variables for the production of reduced acrylamide fried donuts containing different levels of PFE and vitamin $B_{3}$ based on the minimal color indices and acrylamide content, and the highest specific volume and overall acceptability. In order to determine the best PFE to vitamin $\mathrm{B}_{3}$ ratio, the most desired donut formulation with high desirability was selected and proposed as an optimal formulation. It was found that an optimal formulation contained $0.07 \%$ PFE and $1.97 \%$ vitamin $\mathrm{B}_{3}$.

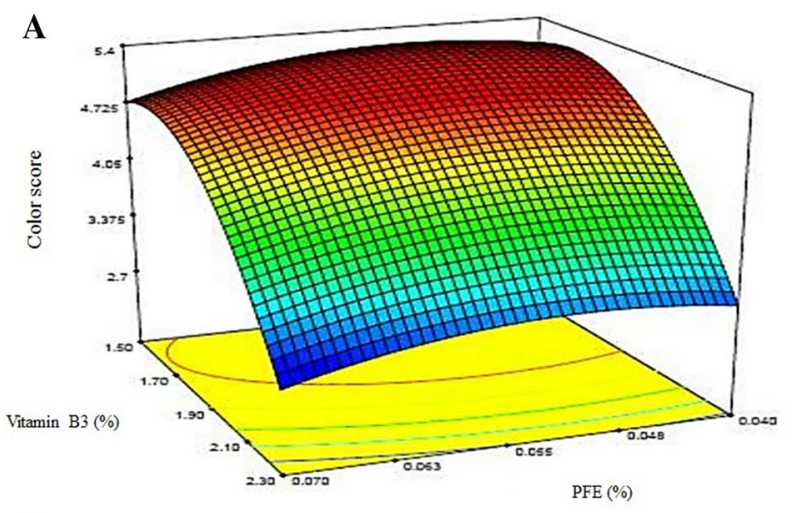

$\mathbf{B}$

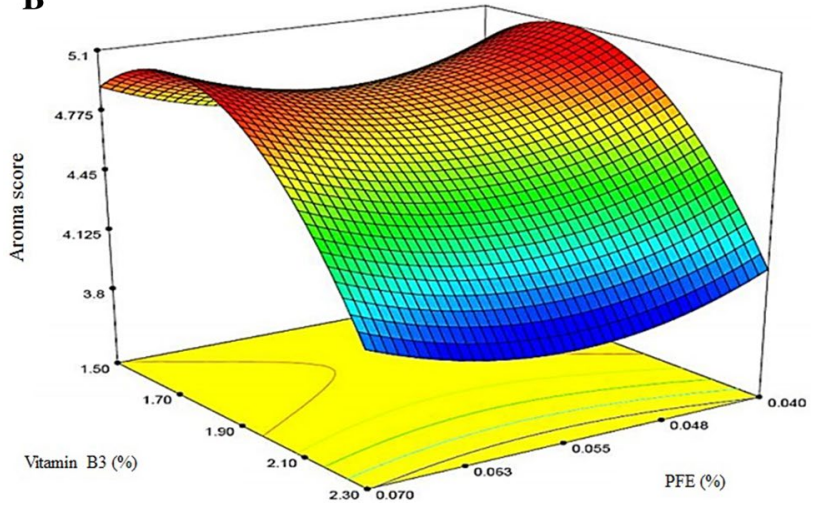

Fig. 8 Response surface plots for the effects of PFE and vitamin $B_{3}$ on donut color (a) and aroma (b) 


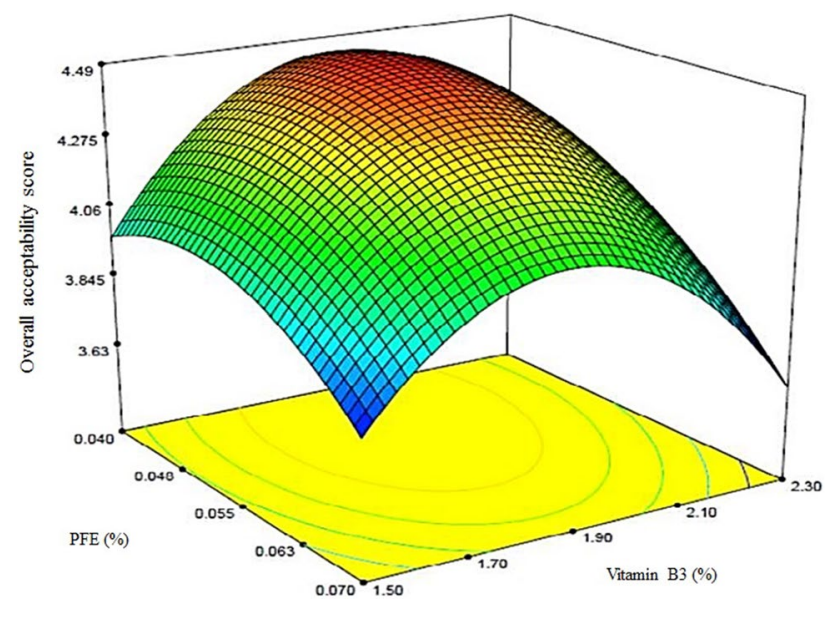

Fig. 9 Response surface plots for the effects of PFE and vitamin $B_{3}$ on donut overall acceptability

\section{Conclusions}

In this study, a reduced acrylamide donut was developed by the addition of pomegranate flower extract and vitamin $\mathrm{B}_{3}$ as potent antioxidants. Response surface methodology was applied to optimize the effects of pomegranate flower extract and vitamin $\mathrm{B}_{3}$ on acrylamide content and physicochemical, textural and sensory attributes. The results of optimization revealed that the content of acrylamide in the final product decreased significantly with increasing amount of pomegranate flower extract and vitamin $\mathrm{B}_{3}$. Specific volume of donuts declined as the level of vitamin $\mathrm{B}_{3}$ increased. As well, peroxide index, thiobarbituric acid value and oil adsorption capacity of the product decreased in the presence of independent variables. The addition of vitamin $\mathrm{B}_{3}$ led to an increase in $\mathrm{L}^{*}$ value, while pomegranate flower extract lowered this parameter. All sensory attributes ranked low scores as function of pomegranate flower extract and vitamin $\mathrm{B}_{3}$ levels. It can be concluded from the results of this study that the addition of pomegranate flower extract and vitamin $\mathrm{B}_{3}$ can possess a positive effect on the nutritional value and acrylamide reduction of the donut. Due to its polyphenolic compounds, pomegranate flower extract increases the antioxidant property of the donut. High antioxidative activity and structural stability of vitamin $\mathrm{B}_{3}$ lead to a considerable decrease in acrylamide content of the final product. In general, pomegranate flower extract and vitamin $\mathrm{B}_{3}$ can be used as food-grade antioxidants and an alternative to synthetic preservatives to reduce acrylamide content in high-temperature treated food products.

Acknowledgements Financial supports from Islamic Azad University, Shahr-e-Qods Branch (Tehran, Iran) are gratefully acknowledged.
Open Access This article is distributed under the terms of the Creative Commons Attribution 4.0 International License (http://creativeco mmons.org/licenses/by/4.0/), which permits unrestricted use, distribution, and reproduction in any medium, provided you give appropriate credit to the original author(s) and the source, provide a link to the Creative Commons license, and indicate if changes were made.

\section{References}

1. G. Budryn, D. Żyżelewicz, E. Nebesny, J. Oracz, W. Krysiak, Influence of addition of green tea and green coffee extracts on the properties of fine yeast pastry fried products. Food Res. Int. 50, 149-160 (2013)

2. K.J. Tan, G.S. Mittal, Physicochemical properties changes of donuts during vacuum frying. Int. J. Food Prop. 9, 85-98 (2006)

3. J. Keramat, A. LeBail, C. Prost, M. Jafari, Acrylamide in baking products: a review article. Food Bioprocess Technol. 4, 530-543 (2011)

4. Y. Liu, P. Wang, F. Chen, Y. Yuan, Y. Zhu, H. Yan, X. Hu, Role of plant polyphenols in acrylamide formation and elimination. Food chem. 186, 46-53 (2015)

5. A. Munin, F. Edwards-Lévy, Encapsulation of natural polyphenolic compounds; a review. Pharmaceutics 3, 793-829 (2011)

6. S. Fernández, L. Kurppa, L. Hyvönen, Content of acrylamide decreased inpotato chips with addition of a proprietary flavonoid spice mix (Flavomare $\left.{ }^{\circledR}\right)$ infrying. Innov. Food Technol. 18, 24-26 (2003)

7. Y. Zhang, W. Xu, X. Wu, X. Zhang, Y. Zhang, Addition of antioxidant from bamboo leaves as an effective way to reduce the formation of acrylamide in fried chicken wings. Food Addit. Contam. 24, 242-251 (2007)

8. L. Marková, Z. Ciesarová, K. Kukurová, H. Zieliński, M. Przygodzka, A. Bednáriková, P. Šimko, Influence of various spices on acrylamide content in buckwheat ginger cakes. Chem Pap. 66, 949-954 (2012)

9. M. Viuda-Martos, J. Fernández-López, J.A. Pérez-Álvarez, Pomegranate and its many functional components as related to human health: a review. Compr. Rev. Food Sci. Food Saf. 9, 635-654 (2010)

10. S. Sreekumar, H. Sithul, P. Muraleedharan, J.M. Azeez, S. Sreeharshan, Pomegranate fruit as a rich source of biologically active compounds. Biomed. Res. Int. 2014, 1-12 (2014)

11. X. Zeng, K.W. Cheng, Y. Jiang, Z.X. Lin, J.J. Shi, S.Y. Ou, F. Chen, M. Wang, Inhibition of acrylamide formation by vitamins in model reactions and fried potato strips. Food Chem. 116, 34-39 (2009)

12. J.F. Vélez-Ruiz, M.E. Sosa-Morales, Evaluation of physical properties of dough of donuts during deep-fat frying at different temperatures. Int. J. Food Prop. 6, 341-353 (2003)

13. F.F. Shih, K.W. Daigle, E.L. Clawson, Development of low oiluptake donuts. J Food Sci. 66, 141-144 (2001)

14. AACC International, Approved Methods of the Association of Cereal Chemists International, 11th edn. (AACC, St. Paul, 2000)

15. Z. Shi, H. Zhang, X. Zhao, Ultrasonic-assisted precolumn derivatization-HPLC determination of acrylamide formed in Radix Asparagi during heating process. J. Pharm. Biomed. Anal. 49, 1045-1047 (2009)

16. M. Jezussek, P. Schieberle, A new LC/MS-method for the quantitation of acrylamide based on a stable isotope dilution assay and derivatization with 2-mercaptobenzoic acid. Comparison with two GC/MS methods. J. Agric. Food Chem. 51, 7866-7871 (2003)

17. AOCS, Official Methods and Recommended Practices of the American Oil Chemists' Society. 4th ed. (AOCS, Champaign, 1997) 
18. AOAC, Official Methods of Analysis. (Association of Official Analytical Chemists, Washington, DC, 1990)

19. J.R.S. Gacula, Statistical Methods in Food and Consumer Research (Academic Press Inc, Cambridge, 1984), pp. 360-366

20. S. Urbančič, M.H. Kolar, D. Dimitrijević, L. Demšar, R. Vidrih, Stabilisation of sunflower oil and reduction of acrylamide formation of potato with rosemary extract during deep-fat frying. LWT Food Sci. Technol. 57, 671-678 (2014)

21. Z. Ciesarová, M. Suhaj, J. Horváthová, Correlation between acrylamide contents and antioxidant capacities of spice extracts in a model potato matrix. J. Food Nutr. Res. 47, 1-5 (2008)

22. M.I. Gil, F.A. Tomás-Barberán, B. Hess-Pierce, D.M. Holcroft, A.A. Kader, Antioxidant activity of pomegranate juice and its relationship with phenolic composition and processing. J. Agric. Food Chem. 48, 4581-4589 (2000)

23. A. Tehranifar, M. Zarei, Z. Nemati, B. Esfandiyari, M.R. Vazifeshenas, Investigation of physico-chemical properties and antioxidant activity of twenty Iranian pomegranate (Punica granatum L.) cultivars. Sci Hort. 126, 180-185 (2010)

24. I. Izzreen, A. Noriham, Evaluation of the antioxidant potential of some Malaysian herbal aqueous extracts as compared with synthetic antioxidants and ascorbic acid in cakes. Int. Food Res. J. 18, 583-587 (2011)

25. S. Pezeshk, M. Rezaei, H. Hosseini, Effects of turmeric, shallot extracts, and their combination on quality characteristics of vacuum-packaged rainbow trout stored at $4 \pm 1{ }^{\circ} \mathrm{C}$. J. Food Sci. 76, 387-391 (2011)

26. M.N. Al-Muammar, F. Khan, Obesity: the preventive role of the pomegranate (Punica granatum). Nutrition 28, 595-604 (2012)

27. D. Bopitiya, T. Madhujith, Efficacy of pomegranate (Punica granatum L.) peel extracts in suppressing oxidation of white coconut oil used for deep frying. Trop. Agric. Res. 25, 298-306 (2015)
28. C. Dobarganes, G. Márquez-Ruiz, J. Velasco, Interactions between fat and food during deep-frying. Eur. J. Lipid Sci. Technol. 102, 521-528 (2000)

29. B. Aydenız, E. Yilmaz, Performance of different natural antioxidant compounds in frying oil. Food Technol. Biotechnol. 54, 21-30 (2016)

30. M.L. Sudha, V. Baskaran, K. Leelavathi, Apple pomace as a source of dietary fiber and polyphenols and its effect on the rheological characteristics and cake making. Food Chem. 104, 686-692 (2007)

31. C.M. Ajila, K. Leelavathi, U.P. Rao, Improvement of dietary fiber content and antioxidant properties in soft dough biscuits with the incorporation of mango peel powder. J. Cereal Sci. 48, 319-326 (2008)

32. M. Majzoobi, S. Sharifi, B. Imani, A. Farahnaky, The effect of particle size and level of rice bran on the batter and sponge cake properties. J. Agric. Sci. Technol. 15, 1175-1184 (2013)

33. R. Wang, W. Zhou, H.H. Yu, W.F. Chow, Effects of green tea extract on the quality of bread made from unfrozen and frozen dough processes. J. Sci. Food Agric. 86, 857-864 (2006)

34. Z. Sheikholeslami, M. Karimi, M. Ghafeh Davoodi, B. Sahraiyan, F. Naghipour, S. Madani, Evaluation of qualitative, visual and sensory properties of cake containing native gum and natural emulsifier. J. Food Sci. Technol. 68, 237-249 (2017)

35. T.M. Lu, C.C. Lee, J.L. Mau, S.D. Lin, Quality and antioxidant property of green tea sponge cake. Food Chem. 119, 1090-1095 (2010)

36. D. Li, Y. Chen, Y. Zhang, B. Lu, C. Jin, X. Wu, Y. Zhang, Study on mitigation of acrylamide formation in cookies by 5 antioxidants. J. Food Sci. 77, C1144-C1149 (2012) 